\title{
A ESCUTA CLÍNICA: UM INSTRUMENTO DE INTERVENÇÃO DO PSICÓLOGO EM DIFERENTES CONTEXTOS
}

\author{
Ana Aparecida N. M. Braga Escola Bahiana de Medicina e Saúde Pública
}

\author{
Mônica Ramos Daltro Escola Bahiana de Medicina e Saúde Pública
}

\author{
Carlos Alberto Ferreira Danon Escola Bahiana de Medicina e Saúde Pública
}

\begin{abstract}
Resumo
O presente artigo discute o impacto da experiência de atendimento clínico na formação do psicólogo a partir da disciplina Diagnóstico e Intervenções, que consta de atendimentos clínicos de psicodiagnóstico, realizados no Serviço Escola de uma instituição provada de ensino superior. O estudo põe em evidência a escuta psicológica clínica como instrumento fundamental ao fazer do psicólogo. Seu objetivo é descrever a relação que os estudantes, egressos da disciplina e também já do curso, fazem entre a prática desenvolvida e a formação de psicólogo. Trata-se de um estudo descritivo qualitativo realizado com 10 ex-alunos da instituição formados entre 2008 e 2010 . Foi aplicado, ao longo de 2011, um questionário semiestruturado submetido a uma análise de conteúdo posterior. Conclui-se que a escuta clínica trabalhada em um processo de psicodiagnóstico é de fundamental importância para a formação do psicólogo, em qualquer campo de atuação, não necessariamente no consultório, pois através dela compreende-se o sujeito e sua questão. Faz-se necessário, portanto, ao psicólogo, ocupar um lugar específico que lhe permita escutar a subjetividade implicada nas diferentes experiências do sujeito, como possibilidade de fazer a diferença nas diversas áreas de trabalho.
\end{abstract}

Palavras-chave: Psicodiagnóstico; Escuta; Psicólogo.

\section{CLINICAL LISTENING: AN INSTRUMENT OF INTERVENTION OF PSYCHOLOGISTS IN DIFFERENT CONTEXTS}

\begin{abstract}
This article discusses the impact of experience of clinical assistance in formation of the psychologists from the discipline Diagnosis and Interventions, which consists of clinical care of psychodiagnostic, made on the Service School of proved an institution of higher education. The study highlights the psychological clinic listening as a fundamental tool for the office of the psychologist. Its goal is to describe the relationship that students - former students of the discipline and also of the course - make between the developed practice and formation of psychologists. This is a qualitative descriptive study conducted with 10 former students of the institution formed between 2008 and 2010. It was applied, throughout 2011, a semi-structured questionnaire submitted to a later content analysis. We conclude that the clinical listening crafted in a psychodiagnostic process is of fundamental importance to the formation of the psychologist, in any field, not necessarily in the office, because through it one understands the subject and its question. It is necessary, therefore, to the psychologist, to occupy a specific place that allows him/her to listen to the subjectivity involved in the different experiences of the subject, as a possibility to make a difference in the various work areas.
\end{abstract}

Keywords: Psychodiagnosis; Listening; Psychologist. 


\section{INTRODUÇÃO}

Diagnóstico é um termo que vem do grego diagnõstikós, que significa conhecer, discernir. ${ }^{(1)}$ A faculdade de diagnosticar, dada a sua significação, pode ser aplicada, portanto, em qualquer área de conhecimento, seja no senso comum, ou mesmo na ciência, passando pelas artes, religião e filosofia.

Compreendido dessa forma, o diagnóstico é inevitável, pois, sempre que explicitamos nossa compreensão sobre um fenômeno, realizamos um de seus possíveis diagnósticos, isto é, discernimos nele aspectos, características e relações que compõem um todo, o qual chamamos de conhecimento do fenômeno. ${ }^{(1, p .1)}$

Nesse aspecto, existem dois sentidos a partir dos quais podemos classificar o conceito de diagnóstico: o amplo e o restrito. O sentido amplo diz respeito a qualquer movimento de compreensão de uma realidade, para além da ciência. E quando falamos de diagnosticar uma realidade no seu sentido restrito, estamos falando da especificidade do campo científico. Sendo assim, a partir da compreensão de um fenômeno, fundamentado na ciência, faz-se necessário estabelecer de qual referencial científico se está partindo, na medida em que podemos ter vários "olhares" sobre um mesmo ponto. ${ }^{(1)}$

Neste estudo, estamos nos referindo a um trabalho de diagnóstico no sentido restrito à ciência, à ciência psicológica, focalizando o campo do psicodiagnóstico, ou diagnóstico psicológico. Para dar conta de garantir um trabalho clínico consistente, Freud ${ }^{(2)}$ destaca que é fundamental um tripé de variáveis, quais sejam: aprofundamento teórico prévio de base, supervisão de um profissional mais experiente, que vise orientar os trabalhos desenvolvidos, e a própria análise pessoal. No que diz respeito ao trabalho de psicodiagnóstico, podemos tomar a mesma orientação, na medida em que se faz necessário uma compreensão teórica dos conceitos e técnicas inerentes ao trabalho, supervisão e trabalho terapêutico pessoal.

Independentemente da abordagem teórica adotada pelo aluno, ou pela qual o mesmo tenha simpatia, faz-se necessário aprofundamento teórico referente às bases do trabalho de psicodiagnóstico no sentido mais amplo, como nos apresenta Rapport ${ }^{(1)}$ ou Ocampo, ${ }^{(3)}$ por exemplo. É importante enfatizar, portanto, que não se pode perder de vista a necessidade de se ter um bom estudo, e sempre que possível, um trabalho pessoal de acompanhamento terapêutico, desvinculado da escolha teórica se tenha feito. 
Quando nos dispomos a realizar um psicodiagnóstico, presumimos possuir conhecimentos teóricos, dominar procedimentos e técnicas psicológicas. Como são muitas as teorias existentes, e nem sempre convergentes, a atuação do psicólogo em diagnóstico, assim como nas outras funções privativas da profissão, varia consideravelmente. ${ }^{(1, \text { p. } 3)}$

Segundo Rapapport, ${ }^{(1)}$ a avaliação psicodiagnóstica subdivide-se a partir de modelos que refletem a forma de interpretação do conhecimento: o primeiro, fundamenta-se na lógica médica, diagnóstica classificatória, psicométrica e comportamentalista; já no segundo podemos agrupar o humanismo, a psicologia fenomenológica e a psicanálise. E, por fim, tem-se uma terceira possibilidade que é a tentativa de integrar esses dois modelos, trazendo uma certa objetividade na subjetividade.

A disciplina Diagnóstico e Intervenção se organiza a partir dessa lógica integrativa com a apresentação, para os alunos, do que vem a ser este trabalho de diagnóstico, quando tem a possibilidade de se responsabilizar por um paciente num processo que envolve uma série de etapas, iniciando de fato o fazer do psicólogo a partir da relação com o paciente. Essa integração possibilita a utilização de algumas técnicas, permeadas por entrevistas clínicas, favorecendo o processo do aluno de compreensão do sujeito, seu contexto e suas questões. A disciplina Diagnóstico e Intervenções em Psicologia tem uma carga horária de 90 horas, faz parte das disciplinas do oitavo semestre do curso de Psicologia e está presente na matriz do curso.

No curso onde essa disciplina está inserida, os estudantes de psicologia, quando na época do Estágio Específico, têm um espectro bastante amplo de atuação, subdividido em duas ênfases: saúde e trabalho, entre as quais deve ser feita uma escolha pelo aluno. Na ênfase saúde, as possibilidades profissionais se realizam na clínica escola, no hospital, ou em unidades públicas de saúde; já na ênfase trabalho, abarca-se primordialmente a atuação em empresas e consultorias em Recursos Humanos. Entre esses dois universos, caminhos profissionais a serem seguidos, quando da entrada no Estágio Específico, o aluno deve fazer uma escolha e, em escolhendo a vertente clínica, escolhe-se também uma corrente teórico-prática na qual se respaldar e aprofundar, a fim de que se tenha uma base consistente de trabalho, sendo ela psicanálise, humanista, cognitivo comportamental, dentre outras. Sendo assim, muitas são as opções, grande é a diversidade, porém todos estão sob o mesmo significante que é ser psicólogo. Essa escolha, ainda que neste momento da disciplina Diagnóstico e Intervenções deve acontecer de maneira informal, por uma abordagem teórica que fundamente a escolha metodológica na ação psicodiagnóstica, pondo em 
evidência a singularidade de cada um, e mesmo na identificação de cada subagrupamento, distingue o psicólogo na sua formação.

Atento à singularidade da experiência formativa, esse estudo tem como objetivo descrever a relação que estudantes egressos da disciplina fazem entre a prática desenvolvida e a formação de psicólogo.

Utiliza-se da metodologia de estudo descritivo qualitativo realizado com ex-alunos do curso de Psicologia da Bahiana, analisando o conteúdo de 10 questionários aplicados com dois psicólogos referentes a cada semestre, contemplando 2007.2, 2008.1, 2008.2, 2009.1 e 2009.2. Esse questionário foi elaborado com dois itens de marcar e, dois, de escrever, favorecendo a coleta de informações de uma maneira específica sobre o assunto pesquisado e também permitindo que cada um possa expor sua individualidade de maneira a enriquecer a pesquisa. Esse estudo tem como importância fundamental a reflexão sobre a realocação do conteúdo no novo currículo, que vem sendo implantado na instituição, assim como amplia a discussão sobre as competências necessárias à formação do psicólogo.

\section{DISCIPLINA DIAGNÓSTICO E INTERVENÇÕES}

A disciplina Diagnóstico e Intervenções em Psicologia apresenta, no contexto pedagógico, uma peculiaridade, de ser a primeira disciplina a abordar o atendimento clínico propriamente dito na grade curricular. Ministrada no $8^{\circ}$ semestre, com 10 alunos em média, por professor, apresenta uma carga horária semanal de oito horas, sendo que quatro são dedicadas ao trabalho de supervisão com o professor, contemplando principalmente aspectos clínicos, ainda que sempre intercalados com articulações teóricas; e quatro dedicadas à atividade prática, período no qual os alunos abarcam os atendimentos clínicos, bem como os estudos teóricos, conceituais e técnicos necessários à construção de um psicodiagnóstico.

O processo acadêmico se inicia com uma atividade de estudo dirigido e apresentação de seminários, que se caracterizam como uma preparação dos alunos, retomando assuntos teórico/práticos trabalhados ao longo do curso que apresentaram os principais conceitos e técnicas das linhas teóricas de trabalho de um psicólogo, tais como psicanálise, humanismo e cognitivo comportamental. Nessas aulas iniciais também são constituídos os contratos 
pedagógicos, acordos referentes aos aspectos atitudinais do aluno, que envolvem sua inserção no local onde o atendimento realizar-se-á, no Serviço de Psicologia (SEPSI) da instituição. Esse contrato tem como objetivo comprometer o aluno com as regras de funcionamento do SEPSI, mas também contempla, desde a postura do aluno, preenchimento de formulários e elaboração de relatório - seguindo as determinações do Conselho Federal de Psicologia - até o próprio atendimento, a relação com os colegas e demais integrantes da equipe, o cuidado pessoal.

Essa etapa de preparação se processa por cerca de duas a três semanas, a critério do professor que autoriza o aluno a iniciar o atendimento. O psicólogo, responsável pelo SEPSI, encaminha ao professor as fichas de pacientes que estão aguardando atendimento, quer já tenham sido triados, que é o fluxo ideal, quer só tenham preenchido a ficha de inscrição e por quaisquer motivos não tenham passado pela etapa da triagem. E o professor indica o paciente para o atendimento, sempre atento à singularidade do aluno.

$\mathrm{O}$ atendimento se realiza em nove etapas, cuidadosamente acompanhadas pelo professor e equipe técnica do SEPSI:

a) Contato telefônico com o paciente ou seu responsável;

b) Primeira Entrevista;

c) Entrevistas Preliminares (com o paciente e com familiares);

d) Planejamento (escolha e aplicação de técnicas a serem utilizadas);

e) Condução dos Atendimentos;

f) Conclusão Diagnóstica;

g) Devolução do trabalho para o paciente e familiares;

h) Encaminhamento do mesmo, quando necessário, seja para psicoterapia, atendimento psiquiátrico, ou quaisquer outros encaminhamentos que se façam necessários;

i) Elaboração de Relatório

O primeiro contato telefônico acontece após uma leitura da ficha de inscrição preenchida pelo paciente, ou seu responsável, bem como do formulário de triagem, caso tenha sido feita. Envolve atenção ao uso das palavras, tom de voz, atenção ao momento de fazer a ligação e a postura frente ao contato.

Revista Psicologia, Diversidade e Saúde, Salvador, dez. 2012; 1(1): 87-100. 
O primeiro contato presencial e entrevista inicial acontecem no SEPSI e buscam identificar e compreender a demanda do paciente, bem como todo o seu contexto familiar, social, história de vida e situação acadêmico/profisssional. Inclui a atenção à comunicação verbal e não verbal, ao conteúdo apresentado e a forma como se apresenta.

As entrevistas preliminares, que se seguem, constam de uma etapa fundamental do processo, vai variar de estrutura, duração e forma, de acordo com a abordagem escolhida, podendo ser mais ou menos estruturada a depender da escola adotada, mas também do estilo do aluno. Contudo, todas devem cumprir o objetivo de possibilitar ao paciente se colocar, para além do que inicialmente o fez procurar o Serviço de Psicologia, dando possibilidade para que outras questões surjam.

A partir daí, conduz-se o trabalho acadêmico mais direcionado à clínica propriamente dita, fazendo as escolhas das técnicas utilizadas. Quem faz isso é o aluno, com o supervisor, no grupo. Essas técnicas variam, desde a escuta clínica, que permeia todo o trabalho, até o uso de testes psicológicos, passando pelos recursos de jogos, desenhos e materiais diversos que podem se fazer necessários para auxiliar a compreensão do caso clínico, fenômeno sobre o qual se está construindo um diagnóstico, conforme conceituamos no começo do presente trabalho. Vale ressaltar que os testes psicológicos utilizados vão variar conforme a demanda do paciente que está em atendimento, bem como da linha teórica de atendimento já em processo de escolha por parte do aluno.

Ao se conseguir compreender melhor, no aspecto psicológico, o que se passa com o paciente, a partir de quatro a seis encontros em média, com as discussões em supervisão e elaboração sobre o caso clínico, evolui-se para a etapa seguinte, quando se deve dar uma devolução para o paciente, bem como aos seus responsáveis, sobre o processo de trabalho desenvolvido, conclusões construídas e encaminhamentos sugeridos, a depender de cada situação.

Finaliza-se o processo construindo um relatório, onde todos esses passos são apresentados, de forma objetiva e cuidadosa, e encaminhado para algum órgão solicitante, quando for o caso. Esse é um relatório que tem como objetivo formalizar o trabalho de atendimento clínico psicológico feito com o paciente, seguindo o escopo de construção de documento previsto pelo Conselho Federal de Psicologia, que contempla: dados do paciente, queixa inicial, técnicas utilizadas, análise dos atendimentos, síntese diagnóstica, 
conclusão/encaminhamento, com data e as devidas assinaturas, do aluno em formação e do professor supervisor.

Ao longo desse complexo trabalho, o aluno vai fazendo intervenções nos atendimentos e participando ativamente nas supervisões, desenvolvendo um "olhar" sobre o paciente que ele próprio está atendendo, e elaborando hipóteses sobre a construção diagnóstica que está fazendo, que são submetidas à apreciação dos colegas, bem como e, sobretudo, da professora/supervisora, que vai provocando reflexões e questionamentos, a fim de que seja desenvolvida a escuta clínica do aluno na sua prática de trabalho, assunto principal do presente estudo.

\section{METODOLOGIA}

Foram convidados para responder o questionário dez alunos ao todo, sendo dois de cada semestre, totalizando cinco semestres, contemplados no período que foi do segundo semestre de 2007 até o segundo semestre de 2009. A todos eles, no início de abril de 2011, foi inicialmente enviado um e-mail ou telefonema formalizando o convite para participar desse levantamento de informações, conforme Anexo 1.

O questionário (Anexo 2) foi elaborado com duas questões objetivas e duas abertas, favorecendo a coleta de informações de uma maneira específica sobre o assunto pesquisado e também permitindo que cada um pudesse expor sua individualidade de maneira a enriquecer a pesquisa.

Após o aceite, foi enviado, outro e-mail (Anexo 3), com um pequeno texto introdutório, apresentando o questionário.

Os resultados foram tabulados e o conteúdo dos comentários organizados em quatro categorias:

a) Escuta clínica como ferramenta de trabalho;

b) Percepção da individualidade/singularidade do sujeito;

c) Papel do psicólogo - construção da identidade profissional;

d) Primeira experiência prático/profissionalizante - coloca-se em prática a teoria. 
Essas categorias permitiram trabalhar as informações articulando-as com os estudos teóricos pesquisados, pois os comentários se constituíram como material mais rico para alcançar os objetivos propostos pelo trabalho.

\section{RESULTADOS ENCONTRADOS E DISCUSSÃO}

A partir do recebimento do questionário, cada um respondeu individualmente e chegou-se à seguinte tabulação dos dados:

1.Qual a relação que você estabelece entre a disciplina Diagnóstico e Intervenções e a sua formação de psicólogo:
( $90 \%$ ) grande
(10\%) média
( $0 \%)$ pequena

2. Qual a sua área de atuação na psicologia?

Clínica - 50\%

Organizacional - 50\%

Hospitalar - $10 \%$

Comunidade $-10 \%$

Obs.: O percentual total soma mais do que 100 porque algumas pessoas atuam em mais de uma área.

3. A escuta clínica desenvolvida no trabalho de diagnóstico faz diferença em seu trabalho, enriquecendo-o?

Observa-se que $90 \%$ dos sujeitos pesquisados identificam uma relação importante entre a disciplina e a formação profissional. A totalidade deles afirma que a escuta clínica faz diferença e enriquece o trabalho enquanto psicólogo. Faz-se necessário destacar que a prática profissional, conforme os resultados do item dois, apresenta-se diversificada, por vezes tendo mais de uma área de atuação desenvolvida pelo mesmo psicólogo, motivo pelo qual os percentuais extrapolam os $100 \%$.

O questionário contava com um item denominado Comentário que permitia ao psicólogo acrescentar observações sobre o tema de estudo. E esse item se constituiu como a etapa mais rica do estudo, onde foi possível coletar dados importantes analisados. Percebeu-se uma identificação temática entre alguns deles, favorecendo a estratificação em categorias, quais sejam: escuta clínica como ferramenta de trabalho, percepção da individualidade/ singularidade do sujeito, papel do psicólogo, construção da identidade profissional e primeira experiência prático/profissionalizante. Coloca-se, em prática a teoria, conforme apresentaremos a seguir. 


\section{Escuta clínica como ferramenta de trabalho}

Um aspecto importante da escuta clínica é a atenção à singularidade do sujeito, assim com a posição subjetiva que o profissional se encontra em relação a isso, ${ }^{(2)}$ conceito que tem semelhanças, ao mesmo tempo em que se diferencia da atenção flutuante, descrita também por Freud $^{(2)}$ nos seus artigos sobre a técnica.

Nos comentários abaixo, pode-se observar certa abertura na escuta que ultrapassa o objetivo da "tarefa", possibilitando acessar o sujeito que fala, na sua singularidade.

Através da escuta diferenciada, direcionada para as demandas do sujeito, o meu trabalho tornou-se atento, minucioso às questões trazidas pelo paciente ou candidato. No caso dos candidatos de avaliação admissional ou periódica, tornei-me mais atenta às questões omitidas no questionário e às suas reações diante da avaliação, podendo abordá-las durante a entrevista, percebendo a postura e o discurso do candidato. A partir das impressões dessa avaliação global, é possível identificar algumas demandas do sujeito, fazendo encaminhamentos e recomendações. (Sujeito 2)

A percepção da escuta como instrumento de trabalho emerge nos discursos dos psicólogos a partir de uma certa abertura por parte do profissional. Desenvolve-se o raciocínio clínico para além da avaliação diagnóstica em si. Pensando na avaliação como um mecanismo possível de exclusão, foi possível perceber a experiência de construção diagnóstica como um processo de compreensão do sujeito na sua complexidade, como podemos exemplificar na fala descrita abaixo:

Sim, embora a predominância das atividades seja na busca de um perfil adequado para as vagas e no desenvolvimento de novas competências e habilidades, a escuta clínica ajuda na identificação de outras interfaces que podem aparecer nessas ocasiões, permitindo uma identificação de um diagnóstico e um possível encaminhamento para psicoterapia. (Sujeito 4)

\section{Percepção da individualidade/singularidade do sujeito}

A importância do manejo de diferentes instrumentos orienta o psicólogo no uso da escuta ao longo do trabalho. Freud, ${ }^{(2)}$ no clássico texto Sobre o Início do Tratamento muito tratou da 
necessidade de se trabalhar bastante a técnica, exatamente para conseguir saber fazer uso dela. A fala de um dos sujeitos entrevistados demonstra essa questão:

Sim, na medida em que tem permitido identificar e avaliar questões especificas, bem como proporcionar a forma mais eficaz para intervir no paciente que foi diagnosticado, visto ser imprescindível a realização do psicodiagnóstico antes de qualquer intervenção do psicoterapeuta. (Sujeito 6)

A experiência de construção diagnóstica psicológica se mostrou capaz de permitir ao aluno compreender que cada sujeito se mostra diferente e a cada um deve ser preservada essa possibilidade, sem que seja formatado o modo de compreendê-lo. O processo pode ser genérico, mas cada atendimento e, principalmente, cada sujeito é único.

Ajuda no conhecimento do indivíduo através da percepção do potencial, mecanismos de defesa, estrutura e dinâmica de sua personalidade, aspectos centrais da personalidade do indivíduo que são essenciais para a compreensão de seus conflitos e tensões, proporcionando também um diagnóstico diferencial. (Sujeito 8)

\section{Papel do psicólogo - construção da identidade profissional}

Seja no consultório propriamente dito, ou em qualquer outra área de atuação profissional, ao psicólogo se requer um trabalho diagnóstico, a competência de construção compreensiva sobre os sujeitos, ou mesmo sobre os contextos nos quais esteja inserido.

A disciplina Diagnóstico e Intervenções nos remeteu, enquanto alunos, a um exercício mais abrangente do que pode aparentar ter sido: o de refletir acerca do papel do psicólogo enquanto detentor de respostas e do saber diante da sociedade. (Sujeito 10)

A partir do uso de instrumentos psicológicos, bem como do contato direto com o paciente, o aluno pôde se posicionar mais perto do profissional que se vislumbrava.

Considero que esse trabalho foi responsável pela construção de uma identidade profissional, na medida em que todo o aporte teórico conquistado até então, pôde ser experienciado e direcionado a possibilidades de ações clínicas. (Sujeito 1) 


\section{Primeira experiência prático/profissionalizante - coloca-se em prática a teoria}

Outra temática que se mostrou presente em alguns comentários, como de grande relevância para os pesquisados, diz respeito à inserção no mundo da realidade profissional percebida pelo pesquisado a partir da inclusão na disciplina Diagnóstico e Intervenções.

Sim. Considero a disciplina "Diagnóstico e Intervenções" como uma primeira abordagem ou uma preparação para o estágio e, consequentemente, para a vida profissional, portanto de grande relevância, mormente para quem pretende trabalhar na clínica. (Sujeito 3)

A experiência clínica se mostrou necessária e formativa para o aluno trabalhar a teoria antes assimilada.

Esta oportunidade de escuta e intervenção na disciplina "Diagnóstico e Intervenções" foi muito importante durante o curso de graduação. Foi quando tive o meu primeiro contato com um paciente e seus familiares e coloquei em prática tudo o que havia estudado teoricamente, sempre com o suporte da orientadora tirando minhas dúvidas e apontando meus erros. (Sujeito 7)

A formação do psicólogo é ampla e complexa, tem diversos campos de atuação e diversas linhas teóricas possíveis de abordagem. Contudo, antes de começarem os estágios curriculares propriamente ditos, passar pela experiência de atendimento clínico favorece o amadurecimento do aluno, habilitando-o para a prática profissional ao desenvolver, dentre outras competências, a condição da escuta clínica, diferencial do psicólogo, independentemente da sua área de atuação, seja em consultório, em hospital, em empresa ou comunidade, e desvinculada da linha teórica de trabalho, seja psicanalítica, humanista ou cognitivo comportamental.

Analisa-se, a partir da apresentação dos dados encontrados, que, principalmente a partir de alguns comentários feitos, é frequente a relação que se estabelece entre a clínica e o trabalho em consultório. Alguns alunos trazem a ênfase da contribuição da disciplina "Diagnóstico e Intervenções" para o trabalho de atendimento clínico stricto sensu, destacando essa relevância. Contudo, é presente sempre a relação que se estabelece entre a escuta e o trabalhar com gente, em qualquer que seja a área da psicologia. 
Dos percentuais computados, observa-se que houve unanimidade, embora seja pequena a amostra de pesquisa, na relação de enriquecimento do trabalho que ora tem sido desenvolvido profissionalmente, ainda que muitos em atividades fora de consultório, a partir da escuta clínica trabalhada na disciplina "Diagnóstico e Intervenções".

Como diz Mannoni:

Os "testes" são para mim apenas um meio e não um fim. [...] E é, pois, ao discurso do sujeito que, sobretudo, vou prender-me. É por isso que me recuso sempre a estabelecer exames fragmentários. [..] Tudo isso só tem sentido situado numa história. ${ }^{(4, \text { p. } 99)}$

Claro que aqui está sendo dada ênfase ao modelo mais subjetivo da compreensão diagnóstica, de acordo com o que foi apresentado na introdução deste trabalho, mas percebe-se o quanto se tem de ganho profissional, a partir dessa prática.

\section{CONSIDERAÇÕES FINAIS}

A partir dos estudos feitos e da análise dos dados coletados através da aplicação do questionário, pôde-se constatar que a escuta clínica, da forma como é trabalhada na disciplina Diagnóstico e Intervenções, constitui-se um instrumento de intervenção do psicólogo, naquele momento da prática clínica, porém, mais adiante, em diferentes contextos. Compreendeu-se que desenvolver uma construção diagnóstica acerca de um paciente é importante e, talvez possamos nos arriscar a dizer que fundamental, na formação de psicólogos, profissionais que vão lidar com gente, a partir de um lugar diferenciado.

Há, ao longo desse processo de aprendizagem, que é um momento para se ter uma visão mais ampla do ser humano, não somente voltado para o que se apresenta explicitamente, um ganho de possibilidades do fazer do psicólogo, no amadurecimento da sua formação.

O aluno percorre um percurso, desde a entrevista inicial, com o próprio paciente e familiares, expõe-se e discute em grupo de supervisão, constrói e elabora hipóteses diagnósticas sobre o caso clínico, arrisca-se e discute. Conduz o caso, decidindo, com colegas e professor supervisor, técnicas a serem utilizadas e intervenções a serem feitas, colocando-se de maneira implicada no processo de forma que se prepara para, e discute após, mas o durante é com o próprio, já "psicólogo", em formação. 
Relaciona-se com a dinâmica institucional, com a divisão de espaços profissionais, com o preenchimento de formulários e cumprimentos de regras. Aprende-se com as variáveis do próprio processo e também com as dos colegas. Ao final, conclui-se o trabalho proposto, estabelecendo encaminhamentos elaborando relatório, de acordo já com as orientações do Conselho Profissional.

Destaca-se, com o presente trabalho, a importância da escuta clínica latu senso, inicialmente desenvolvida em atendimento clínico propriamente dito, para a atuação do psicólogo, trabalhando ele em consultório, hospital, empresa, comunidade, ou qualquer outro espaço.

\section{REFERÊNCIAS}

1. Rappaport CR. Temas básicos de psicologia. São Paulo: EPU; 1996.

2. Freud S. Edição standard brasileira das obras psicológicas completas de Sigmund Freud. Rio de Janeiro: Imago; 1977.

3. Ocampo MLS. O processo psicodiagnóstico e as técnicas projetivas. São Paulo: Martins Fontes; 2001.

4. Mannoni M. A primeira entrevista em psicanálise. Rio de Janeiro: Campus; 1980.

\section{ANEXO 1:}

"Queridos colegas psicólogos, ex-alunos da disciplina Diagnóstico e Intervenções.

Estou escrevendo um artigo cujo título provisório é "Psicodiagnóstico - Um processo importante na formação do psicólogo", onde discorro sobre a idéia de que, independente da área de atuação do psicólogo, a escuta clínica é fundamental para a diferenciação profissional.

Para tanto, preciso aplicar um questionário, com poucas perguntas, com psicólogos já formados, que passaram por mim nesta disciplina, motivo pelo qual envio este $e$-mail para vocês. O quanto antes preciso do retorno de cada um de vocês, por e-mail, ou por telefone: (71)3353-1742 / (71)9979-8384.

Um abraço,

Ana Aparecida N. Martinelli Braga CRP 03/01763" 


\section{ANEXO 2:}

1. Qual relação que você estabelece entre a disciplina "Diagnóstico e Intervenções" e a sua formação de psicólogo?
( ) grande
( ) média
( ) pequena

2. Qual a sua área de atuação profissional na psicologia?

3. A escuta clínica desenvolvida por você no trabalho de diagnóstico e intervenção faz diferença em seu trabalho hoje?
( ) muita
( ) pouca
( ) nenhuma

Comente

ANEXO 3:

"Caros ex-alunos, hoje psicólogos, gostaria, antes de mais nada, de agradecer a sua presteza em contribuir para este relato de experiência que está sendo desenvolvido no artigo "Psicodiagnóstico Um processo importante na formação do psicólogo?"

Solicito que responda as perguntas abaixo e me envie o mais breve possível. Seu nome será mantido em sigilo e tão logo eu conclua o trabalho, enviarei cópia do mesmo na íntegra.

Um abraço,

Ana Aparecida Braga

CRP.03/01763...."

Revista Psicologia, Diversidade e Saúde, Salvador, dez. 2012; 1(1): 87-100. 